\title{
Radionuclide imaging in risk assessment after acute coronary syndromes
}

\author{
J E Udelson, E J Flint
}

Heart 2004;90(Suppl V):v16-v25. doi: 10.1136/hrt.2004.041194

$\mathrm{T}$ e National Service Framework for coronary artery disease $^{1}$ acknowledged a role for myocardial perfusion imaging (MPI) in diagnosis and risk assessment of angina and quoted the cost-effectiveness data from the EMPIRE study. ${ }^{2}$ One of its most important effects, however, was to imply increased cardiological management for patients with acute coronary syndromes (ACS). The joint British Cardiac Society and Royal College of Physicians' guidelines for the management of patients with ACS without persistent ECG ST segment elevation ${ }^{3}$ have recommended evaluation of chest pain assessment units based on evidence both from the USA ${ }^{4}$ and $U^{5}$ for patients at low cardiac risk. High risk patients, including those with raised troponin, recurrent ischaemic symptoms and/or ST segment changes or adverse stress test results, should have urgent coronary angiography with intention of revascularisation to improve outcomes. In patients with confirmed ACS without recurrent spontaneous symptoms, stress testing provides valuable risk stratification, ${ }^{6}$ with pharmacological stress enabling assessment of those who cannot exercise. ${ }^{7}$

This article will review the role of radionuclide techniques across the spectrum of patients with ACS, from those presenting with suspected ACS but without diagnostic initial ECG changes, to the now well defined syndromes of unstable angina (UA)/non-ST segment elevation myocardial infarction (NSTEMI) and ST segment elevation myocardial infarction (STEMI). In these settings, radionuclide imaging techniques have a unique role, strongly supported by an evidence base, as contemporary imaging techniques supply simultaneous information on stress and rest perfusion as well as left ventricular (LV) function. Risk assessment together with appropriate aggressive secondary prevention is particularly helpful in the UK situation of uneven access to revascularisation, allowing for appropriate prioritisation of patients.

\section{IMAGING IN THE CHEST PAIN ASSESSMENT UNIT, EMERGENCY DEPARTMENT, AND MEDICAL ASSESSMENT UNIT}

Patients with symptoms suggestive of acute myocardial ischaemia but with non-diagnostic ECG and negative 12 hour enzymes are often subsequently stress tested, mostly with negative results, enabling discharge. ${ }^{4}$ Thus, there are a substantial number of unnecessary hospitalisations and unnecessary resource utilisation. The required 16-24 hour observation and testing period may be significantly shortened by MPI. The feasibility of imaging in this situation was originally demonstrated in the Netherlands by Wackers and colleagues $^{8}$ using planar thallium-201 techniques. The rapid redistribution characteristics of thallium-201, its limited acute availability, and the need for portable camera systems made widespread use impractical. Many of these impediments to application of imaging in this setting were overcome by the introduction of the technetium 99m (Tc 99m) based agents, sestamibi and tetrofosmin. With their relative lack of redistribution, images may be acquired 45-60 minutes after injection, ${ }^{9}$ and reflect myocardial blood flow at the time of injection in the chest pain assessment unit (CPAU). High quality single photon emission computed tomographic (SPECT) imaging may then be undertaken in the nuclear medicine department.

\section{Relationship of imaging results and outcomes}

There is now a substantial body of literature evaluating rest SPECT perfusion imaging in the emergency setting. Bilodeau and colleagues ${ }^{10}$ performed rest SPECT sestamibi imaging in patients already hospitalised for suspected UA, injecting them with tracer at the time of an episode of spontaneous chest pain while also recording an ECG. The sensitivity of the SPECT sestamibi images for determining the presence of a severe coronary stenosis on subsequent angiography was $96 \%$, while the sensitivity of ECG was only $35 \%$. In patients with acute myocardial infarction (MI), a perfusion defect involving as much as $20 \%$ of the left ventricle can exist on acute rest sestamibi imaging in the presence of a normal or non-diagnostic ECG. ${ }^{11}$ Despite the presence of resting wall motion abnormalities on echocardiography being less sensitive than resting perfusion defects in the diagnosis of ACS, the lower availability of emergency MPI in most European countries may result in the suboptimal, pragmatic use of ultrasound. Varretto and colleagues ${ }^{12}$ first reported from Italy that over 18 months follow up in patients with suspected ACS but a normal resting SPECT sestamibi study in the emergency department (ED), there were no untoward cardiac events. A high negative predictive value for ruling out MI has equalled or exceeded $99 \%$ in all published series. ${ }^{12-15}$ Patients with positive results have a substantially higher risk of untoward cardiac events during the index hospitalisation as well as during follow up (figs 1 and 2). Such data suggest that MPI provides important information to assist triage decisions (admit or not admit) in the ED. Where cases of negative resting MPI arise, it is important to follow up with stress testing, secondarily performed to assess or discard the diagnosis of coronary artery disease.

\section{Comparison with enzymatic and biomarkers of ACS}

There is an important distinction between the information provided by perfusion imaging and that from biomarker enzyme analysis of myonecrosis. Perfusion imaging data should be abnormal almost immediately after an abnormality in myocardial blood flow is established, while markers may require up to $12-18$ hours after symptom onset for optimal sensitivity. Moreover, MPI data should theoretically be abnormal whenever myocardial blood flow is abnormal (that is in both UA and acute MI), whereas biomarkers are positive in virtually all patients with acute MI, but only approximately

Abbreviations: ACS, acute coronary syndromes; BMIPP, $\beta$-methyliodophenyl pentadecanoic acid; CPAU, chest pain assessment unit; ED, emergency department; LV, left ventricular; MAU, medical assessment unit; MIBG, I-123 metaiodobenzylguanidine; MI, myocardial infarction; MPI, myocardial perfusion imaging; NSTEMI, non-ST segment elevation myocardial infarction; PTCA, percutaneous transluminal coronary angioplasty; STEMI, ST segment elevation myocardial infarction; SPECT, single photon emission computed tomographic; Tc $99 \mathrm{~m}$, technetium 99m; UA, unstable angina 


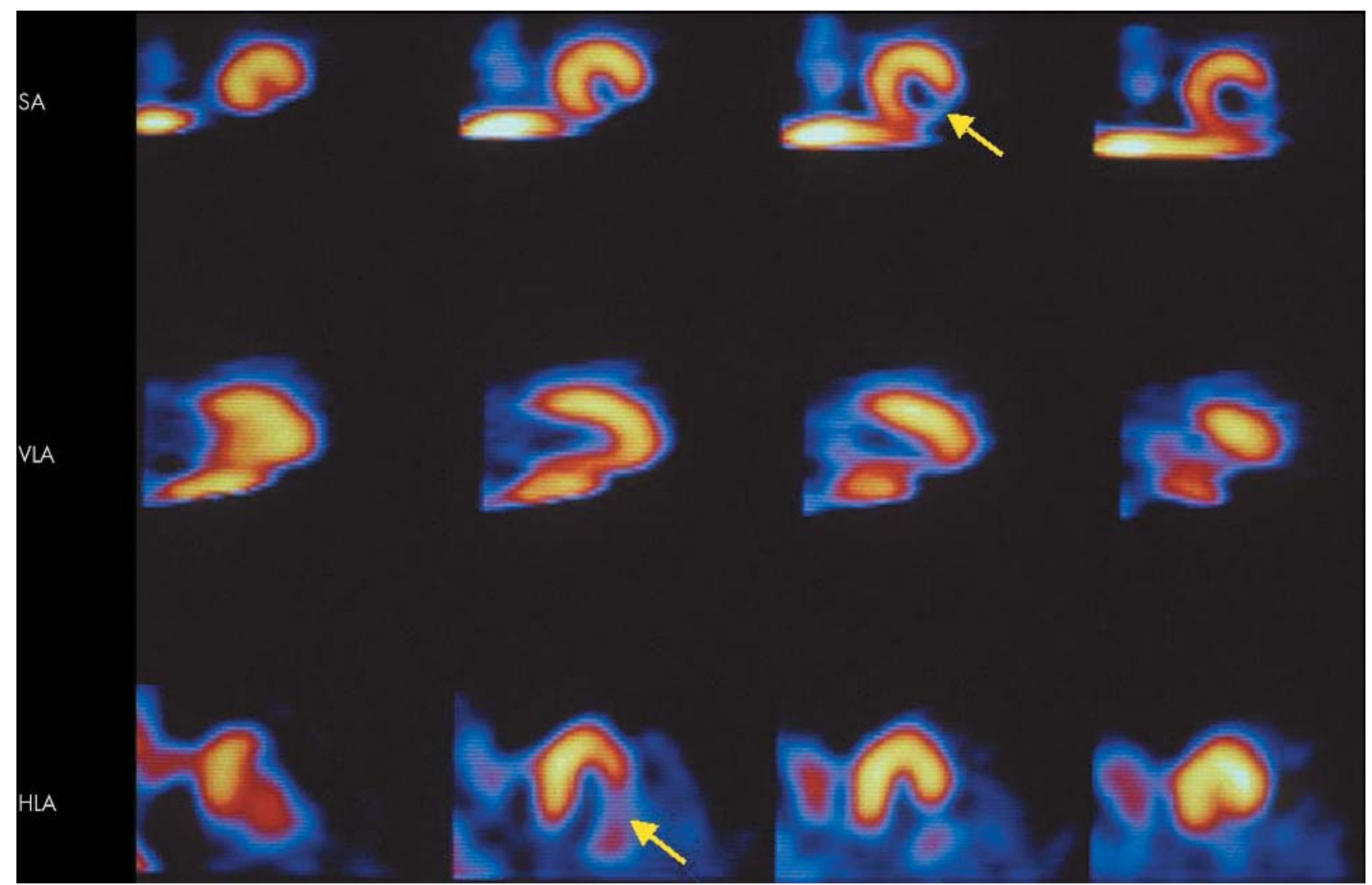

Figure 1 SPECT resting Tc $99 \mathrm{~m}$ sestamibi imaging of a 39 year old man who presented to the ED with chest pain, atypical for angina and a near normal initial ECG. The images demonstrate a severe resting perfusion defect in the inferolateral wall. As a result of these findings and of ongoing symptoms, he was taken to the catheterisation laboratory, where an acute left circumflex occlusion was found and treated with primary angioplasty.

$30-35 \%$ of those with a clinical syndrome of UA. Consistent with these concepts, Kontos and colleagues ${ }^{16}$ found SPECT sestamibi performed in the ED $92 \%$ sensitive for detecting acute MI, while initial cardiac troponin I values drawn at the same time had a sensitivity of only $39 \%$. Subsequently, the maximum troponin I over the first 24 hours had sensitivity similar to that of the acute rest sestamibi imaging, but at a distinctly later time point. Thus, acute MPI has the potential to identify ACS much earlier in their evolution than enzyme markers. Moreover, SPECT perfusion imaging data have been shown to provide incremental risk stratification value for predicting unfavourable cardiac events (fig 3). ${ }^{15}$

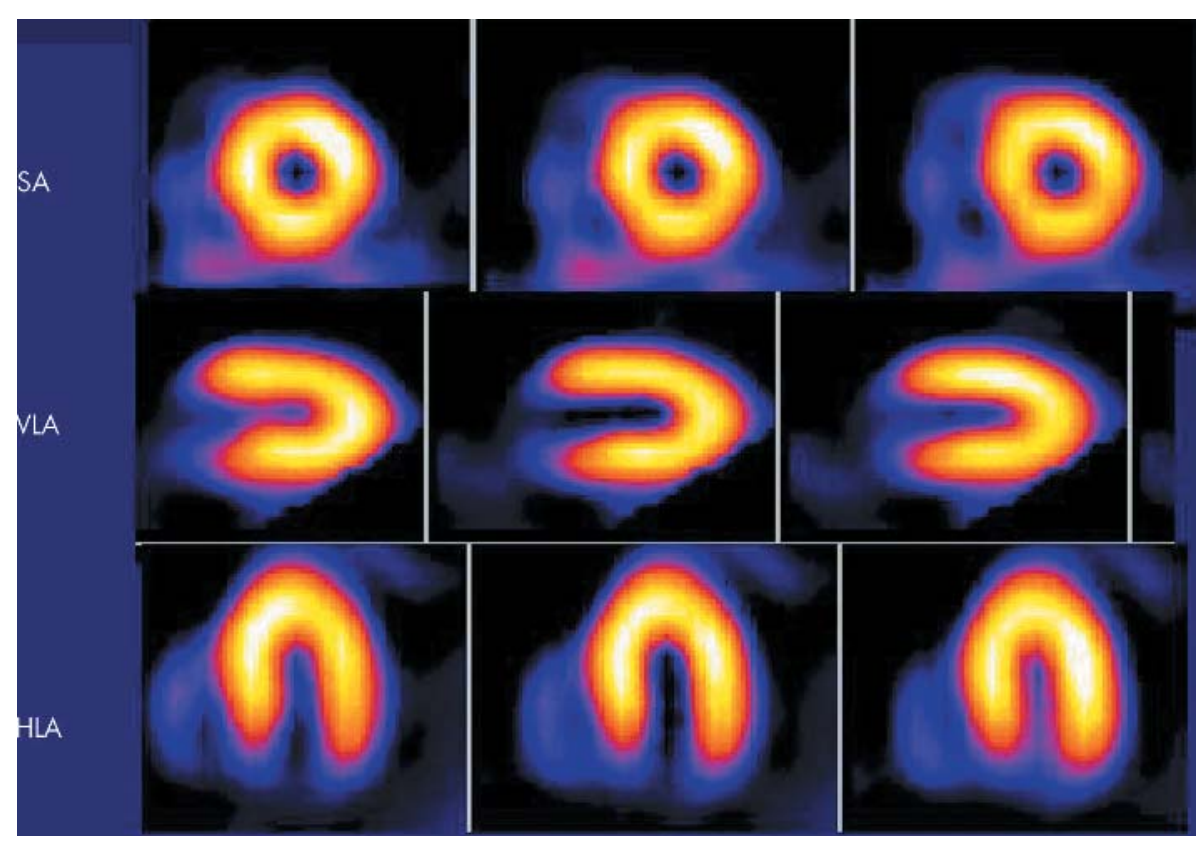

Figure 2 Short axis, vertical and horizontal long axis SPECT images of a 52 year old man who presented to the ED with chest pain atypical for angina and a initial ECG with non-specific ST segment abnormalities not diagnostic for acute ischaemia. He was injected with tc $99 \mathrm{~m}$-sestamibi at rest in the ED and underwent SPECT imaging in the nuclear cardiology laboratory soon thereafter. The images show a completely normal resting perfusion pattern, and the gated SPECT imaging of resting LV function (not shown) was also normal. This finding is associated with a very low probability of $\mathrm{MI}$ and acute ischaemic syndrome, suggesting that such a patient may be discharged directly from the ED. 


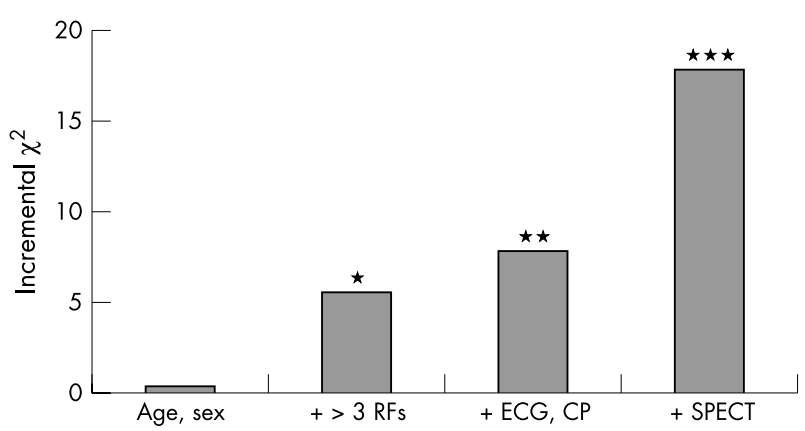

Figure 3 Analysis of the incremental value of resting perfusion imaging data to predict cardiac events in ED patients with suspected ischaemia. The incremental $\chi^{2}$ value (y axis) measures the strength of the association between individual factors added to the knowledge base in incremental fashion ( $x$ axis) and unfavourable cardiac events. Addition of SPECT perfusion imaging data adds highly significant value even with knowledge of age, sex, risk factors for coronary artery disease, ECG changes and presence or absence of chest pain. Adapted from Heller and colleagues. ${ }^{15}$

\section{Randomised trials involving ED patients with acute chest pain and MPI}

Stowers and colleagues ${ }^{17}$ evaluated 46 patients presenting to the ED with ongoing chest pain and a non-diagnostic ECG before acute MPI. The patients who were randomly assigned to a perfusion imaging guided strategy compared to a conventional strategy incurred costs of $\$ 1843$ less, with shorter median hospital stay and shorter median intensive care stay, and fewer cardiac catheterisations, but no difference in outcome at 30 days. These data suggest that similar outcomes could be achieved with lower resource utilisation using an MPI guided strategy.

The ERASE chest pain trial ${ }^{18}$ randomised 2475 patients with symptoms suggestive of ACS presenting to a diverse group of hospital EDs to a usual evaluation strategy or a strategy including supplementary information from acute rest SPECT sestamibi imaging. Imaging results were immediately incorporated into the triage decision in the scan strategy group. There was a highly significant $20 \%$ relative reduction in risk of unnecessary admissions of patients ultimately found not to have an acute ischaemic syndrome $(\mathrm{n}=2127$, OR $0.68, \mathrm{p}<0.001)$ in those randomised to the scan strategy. The benefit of more appropriate direct discharge from the ED was observed irrespective of age, sex, risk factors, or relevant imaging experience. In a multivariate analysis, the imaging data were among the most powerful factors associated with the decision to appropriately discharge the patient from the ED.

Thus, the evidence from these strategy controlled, randomised trials suggests that incorporating high quality MPI can improve triage decisions in the ED among patients with suspected ACS, but no definitive ECG changes of acute ischaemia or infarction.

\section{Application of MPI and interpretation in suspected acute ischaemia patients}

Patients who report to the ED with symptoms consistent with an ACS and an ECG diagnostic for acute ischaemia or infarction do not benefit from acute imaging, and are triaged based on the presence of ST elevation or ST depression into appropriate therapy algorithms.. ${ }^{19} 20$ Ideal patients for imaging are those with non-diagnostic or normal ECGs and symptoms suspicious for acute ischaemia, with no prior history of MI or significant Q waves on the ECG. The latter patients will often have a perfusion defect representative of the old infarction, and thus the data will not be as helpful for discrimination of a new acute ischaemic syndrome.
In the UK, the impetus to develop CPAUs in an emergency admission area is supported in the fifth joint report on provision of cardiac services. ${ }^{21}$ In addition to the data on negative predictive value and the favourable impact of MPI on use of resources and clinical decision making, the evidence from Ziffer and colleagues ${ }^{22}$ of a SPECT imaging based chest pain analysis protocol reducing the missed infarction rate from $1.8 \%$ to $0.1 \%$ is encouraging.

While the data from the ERASE chest pain trial and other studies provide compelling evidence for incorporation of acute SPECT MPI into ED evaluation strategies for patients with suspected acute ischaemia, future studies will refine the patient population likely to benefit most from such a procedure. Such patients are likely to have certain clinical characteristics, which may on a larger scale be identified by ECG predictive instruments, for example, in which the pretest probability of acute ischaemia is so low that imaging is not beneficial. A future direction in imaging might find a potential use of new compounds which can trace hypoxic myocardium. The optimal combination of imaging data, enzyme data and stress testing, and the temporal distribution of such testing also need to be better defined. However, given the very high rate of admission for observation or evaluation from the ED of such patients, the incorporation of MPI has the potential to reduce unnecessary hospitalisations significantly, with potential associated cost savings.

\section{ASSESSMENT OF PATIENTS WITH NSTEMI/UA}

While there is general agreement that patients with high risk clinical characteristics (recurrent in-hospital ischaemic symptoms) in the clinical setting of UA should undergo direct and prompt catheterisation, there is less clear consensus regarding patients with intermediate or low clinical risk as originally defined by the US Agency for Health Care and Policy Research guidelines, ${ }^{23}$ including those with medically stabilised UA after initial evidence based therapies. In such patient groups MPI has been shown to identify a low risk outcome group, suggesting that such patients can be managed conservatively without catheterisation (fig 4), ${ }^{24}$ while patients with significant demonstrable inducible ischaemia are at high risk, and are therefore selected for intervention (fig 5). The American Heart Association/ American College of Cardiology guidelines on cardiac radionuclide imaging ${ }^{25}$ considers the use of stress MPI for detecting residual ischaemia and the use of radionuclide angiography to assess LV function in this syndrome as class I indications.

Debate has centred over whether outcomes in all patients with UA/NSTEMI are improved with an early invasive strategy (referral to catheterisation and revascularisation based on anatomic findings at angiography) versus an early conservative strategy (stress testing with referral to catheterisation based on the extent of ischaemia by imaging or on the basis of spontaneous ischaemia). The evidence from several randomised trials is inconsistent on this point, likely reflecting important differences between the studies in patient populations and design features. Strategies using catheterisation in the majority of ACS patients, most often between 4-48 hours after presentation, do not reflect the reality of UK practice where a conservative strategy covers most cases.

Four major clinical trials have now compared outcomes for these two strategies, ${ }^{26-29}$ and based on the results available at the time, the 2000 American College of Cardiology/American Heart Association guidelines assigned both early invasive and early conservative strategies a class I indication. ${ }^{19}$ A recent economic analysis of the VANQWISH study ${ }^{30}$ found that the conservative strategy is preferable on both clinical (worse survival at 1 year of invasive strategy patients) and economic 


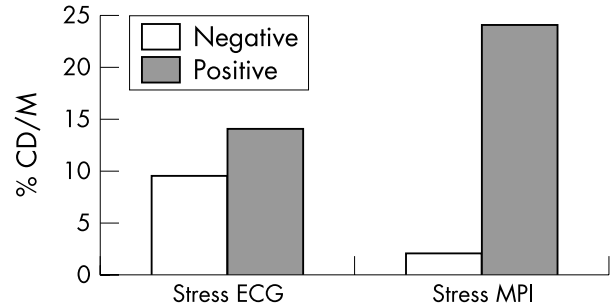

Figure 4 Data supporting the use of MPI as the decision point in a conservative management strategy in patients with non-ST segment elevation ACS. The predictive value of stress MPI and stress electrocardiography (stress ECG) is shown in patients studied after initial stabilisation of UA with medical therapy. This figure summarises the results of three studies in which the incidence of cardiac death or nonfatal $\mathrm{Ml}$ were assessed as end points (\% CD/M on y axis) during follow up after stabilisation of UA. The presence of reversible perfusion defects reflective of ischaemia (positive stress MPI) was strongly predictive of cardiac events in this setting; the absence of inducible ischaemia on MPI (negative stress MPI) identifies a low risk group suggesting that such patients can be managed conservatively. Data are less consistent on the use of exercise electrocardiography in this setting. Adapted from Brown. ${ }^{33}$

grounds. The FRISC-II study ${ }^{28}$ suggested benefit for an invasive approach. However, the early conservative strategy group could only be sent to angiography for potential intervention given a stress test with a very strongly positive result, far more strongly positive than is usually considered as a basis for catheterisation referral (at least in the USA). Thus, the early conservative arm in the FRISC-II trial was likely enriched with high risk patients who may well have benefited from revascularisation, providing an advantage to the early invasive randomisation group. In this regard, a recent analysis using the FRISC-II stress test crossover criteria applied to the VANQWISH population ${ }^{31}$ found a significant population with high risk coronary angiographic features who would not have been sent for catheterisation had the FRISC-II criteria been applied in that study.

The TACTICS-TIMI-18 trial, ${ }^{29}$ in which patients with symptoms of ACS and supportive ECG changes received the platelet inhibitor tirofiban and were randomised to an early invasive or an early conservative strategy, found outcomes favouring the invasive strategy. The absolute benefit was relatively small (reduction of 2 per 1000 deaths and 20 per 1000 non-fatal infarctions), and appeared driven by the clinically higher risk patient subset-that is, the subgroup with elevated troponins and other high risk markers. ${ }^{29}$ The VANQWISH and TACTICS-TIMI-18 studies found similar trends in costs: significantly higher initial hospitalisation costs for early invasive therapy and a partial 50\% recoup of these costs in follow up..$^{32}$

For patients with UA/NSTEMI, a recent meta-analysis has suggested no clear superiority of early revascularisation in ACS. ${ }^{33}$ Such data suggest that a conservative management strategy, in which stabilised patients are risk stratified by stress MPI, offers similar outcomes with fewer invasive procedures. This concept is relevant for UK practice even in the setting of troponin assisted confirmation of ACS and the complexity introduced by the redefinition of $\mathrm{MI}^{34}$ suggested by the joint European Society of Cardiology/American College of Cardiology committee statement. Given the absence of a clear indication to proceed with an aggressive interventional strategy in all patients with ACS, the principles of assessing LV function and reversible ischaemia to guide management strategy remain powerful.

Thus, the use of MPI following ACS on current evidence as the important decision driving component of the early conservative strategy may therefore reduce the trend towards diminishing marginal returns with invasive management. A recent retrospective analysis of the TIMI-III-B data has shown that by using a simple clinical score (based on age, creatine kinase myocardial band (CK-MB), history of accelerated angina and ST depression on the ECG), over half

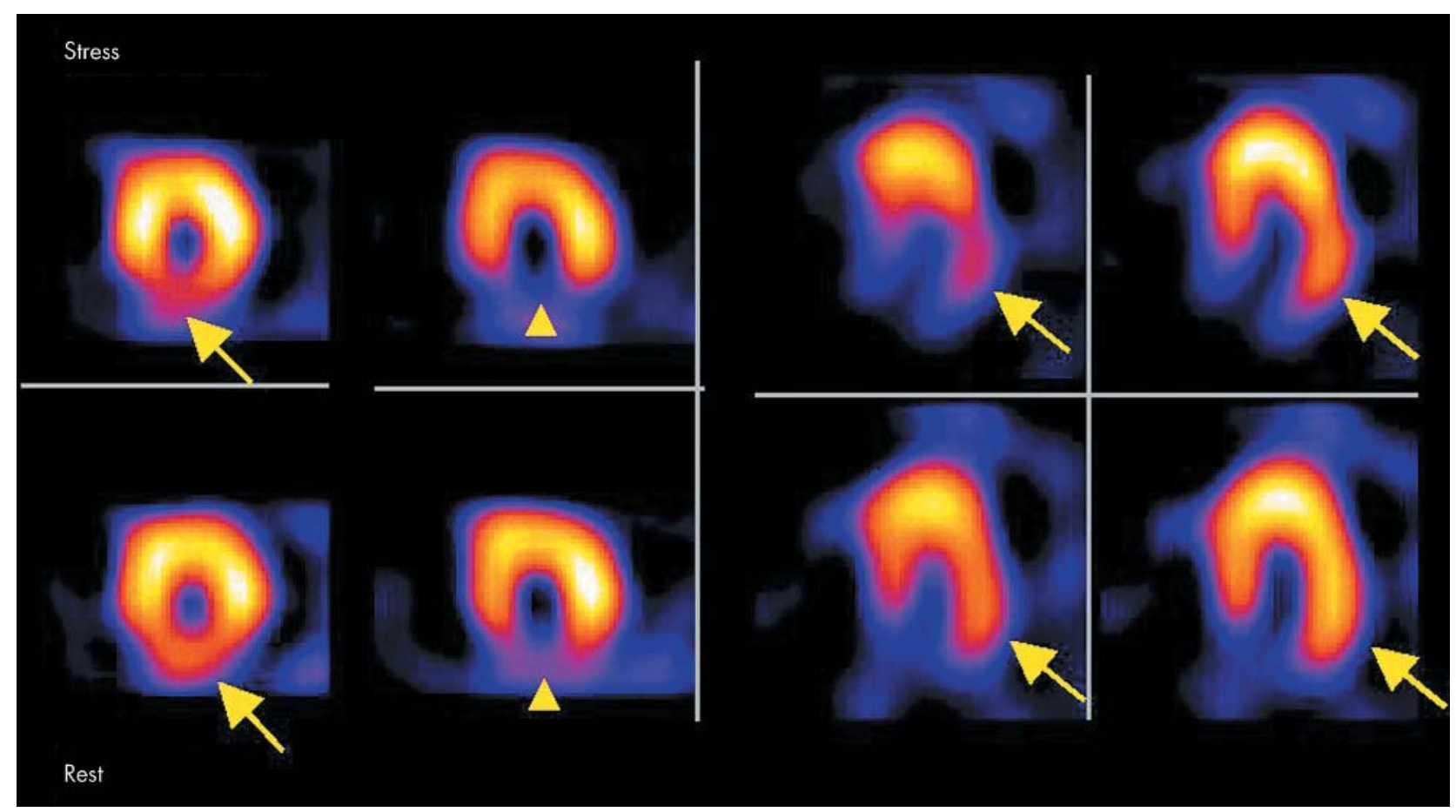

Figure 5 Positive MPI study in a patient with medically stabilised UA. After presentation, symptoms were controlled with initial medical therapy, and stress MPI was performed. There is evidence of an inferobasal infarct (arrowhead) but the large extent of inducible ischaemia involving the inferoapical and lateral walls (arrows) suggests high risk for future events, and the patient was subsequently referred for catheterisation. 
of the population could be classified as low risk. These patients demonstrated no benefit in prevention of death or MI up to one year after the early invasive strategy, ${ }^{35}$ suggesting that for such patients, a conservative strategy incorporating stress MPI would help to optimally select the subgroup most likely to benefit from intervention, as well as those not likely to benefit. In TACTICS-TIMI-18, the troponin positive subgroup, constituting $60 \%$ of the total population, had a larger reduction in death or MI with the early invasive strategy. ${ }^{29}$ Therefore TACTICS type patients without elevation of troponin or high TIMI risk score ${ }^{36}$ may be optimally managed by a more conservative approach with risk stratification by using imaging techniques. The UK guidelines ${ }^{3}$ include the conservative strategy even for patients with an abnormal troponin, providing symptoms have settled for 48 hours and there are no other high risk features.

\section{Importance of LV function assessment in patients with UA}

Most of the randomised trials of invasive versus conservative management strategies in UA/NSTEMI have not clearly taken into account the presence or magnitude of LV dysfunction, the importance of which should not be lost in the debate on the optimal strategy. In most patients with UA/NSTEMI, LV function is usually preserved, and is therefore not as powerful an incremental discriminator of subsequent risk. In a randomised trial of medical versus revascularisation therapy in UA, the degree of LV dysfunction played an important role in defining an advantage of surgical therapy among those whose baseline resting LV ejection fraction was $<50 \% .{ }^{37}$ In the contemporary practice of radionuclide imaging, referral for stress perfusion imaging will almost always result in the provision of simultaneously derived information on LV function, using the now widely available gated SPECT techniques. ${ }^{38}$ This information will more fully inform the decision process than information on perfusion alone.

\section{Future directions}

A potential future approach to risk stratification in patients with suspected ACS or UA/NSTEMI involves the use of imaging fatty acid metabolism. Evidence suggests that following a regional ischaemic insult, abnormalities in fatty acid metabolism resulting from ischaemia may persist long after perfusion has returned to normal, demonstrating the property of ischaemic memory. Imaging fatty acid metabolism may therefore allow assessment of the recent presence and extent of ischaemia even well after symptom resolution, potentially without the need for stress.

\section{Trial acronyms}

- EMPIRE: Economics of Myocardial Perfusion Imaging in Europe

- ERASE Chest Pain: Emergency Room Assessment of Sestamibi for Evaluation of Chest Pain

- FRISC-II: Fast Revascularization during Instability in Coronary artery disease

- TACTICS: Treat Angina with Aggrastat and determine Cost of Therapy with an Invasive or Conservative Strategy

- TIMI: Thrombolysis In Myocardial Infarction

- VANQWISH: Veterans Affairs Non-Q-Wave myocardial Infarction Strategies in-Hospital
In a recent study, Kawai and colleagues used a radiolabelled fatty acid analogue, $\beta$-methyl-iodophenyl pentadecanoic acid (BMIPP) with SPECT imaging, performed 1-5 days after presentation in 111 patients with suspected ACS. The BMIPP images showed greater sensitivity than rest perfusion imaging within 24 hours in identifying the presence and site of the culprit coronary stenosis or spasm (74 $v 38 \%$ respectively, $\mathrm{p}<0.05$ ) at similar high specificity (fig 6). ${ }^{39}$ Future studies will determine whether such a technique can help guide management in such patients.

\section{ASSESSMENT OF PATIENTS WITH STEMI}

Clinical variables such as recurrent ischaemia, heart failure, and non-acute arrhythmias during the hospitalisation for acute STEMI identify a patient subgroup at high risk in which there is general agreement that early catheterisation and intervention is indicated. However, the majority of patients surviving the initial acute infarction period will have a relatively stable and uncomplicated course. In these patients, current guidelines generally recommend noninvasive risk stratification before hospital discharge. ${ }^{20}$

\section{Assessment of myocardial perfusion and inducible ischaemia after acute STEMI}

A large body of literature documents three major determinants of natural history risk following an index acute infarction. These factors include residual resting LV function, the extent of ischaemic, jeopardised myocardium, and the susceptibility to ventricular arrhythmias. Thus, measures of LV function and the extent of inducible ischaemia would be expected to provide important prognostic information in the aftermath of acute MI, with the potential to guide management decisions regarding catheterisation and subsequent intervention. Gated SPECT imaging, on the basis of its comprehensive ability to provide all of this important information, has the potential to be the single most important test in the stable patient following a STEMI.

One of the earliest studies to examine the relation of perfusion imaging data to outcomes in stable patients following MI was published by Gibson and colleagues in 1983. ${ }^{40}$ In this report, thallium 201 scintigraphic data contained the most robust information on stratifying risk, in that a low risk thallium 201 image (defined as one thallium defect, no reversible defects or no lung uptake) was associated with a very low risk natural history outcome, with only $6 \%$ of patients suffering cardiac events during a three year follow up. In contrast, patients with a low risk exercise test (defined as no ST depression or exercise induced angina) had a $25 \%$ incidence of cardiac events on follow up, while patients with a low risk coronary angiogram (defined as $0-1$ vessel disease) had a $22 \%$ incidence of subsequent events.

As data on the value of exercise perfusion scintigraphy was emerging throughout the early to mid 1980s, a simultaneous line of investigation was being reported on the value of pharmacologic stress imaging in the same clinical setting. An important proportion of patients following uncomplicated MI are not able to exercise even to a submaximal workload. These patients are generally at higher risk of subsequent cardiac death or MI compared to the cohort able to exercise. ${ }^{41}$ Leppo and colleagues ${ }^{42}$ reported that the presence of redistribution (ischaemia) on a dipyridamole thallium 201 scan was the only significant predictor of important cardiac events on multi-variable analysis, while the absence of redistribution defects identified a low risk cohort. Subsequent reports confirmed these findings. ${ }^{43} 44$

Many studies in the thrombolytic era have reported similar favourable results regarding the relation of stress induced scintigraphic ischaemia to outcomes, as those reported in the pre-thrombolytic era. Travin and colleagues ${ }^{45}$ used Tc $99 \mathrm{~m}$ 


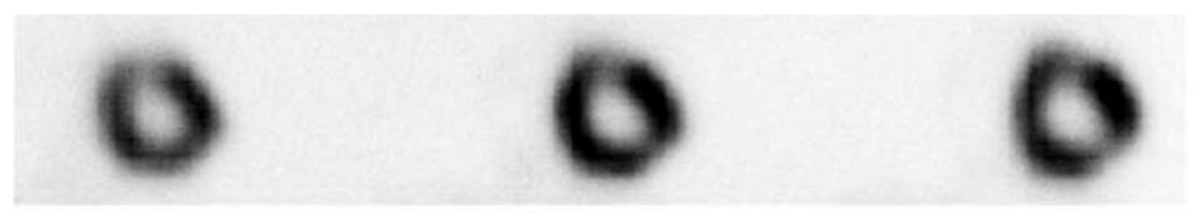

Rest thallium-201 SPECT

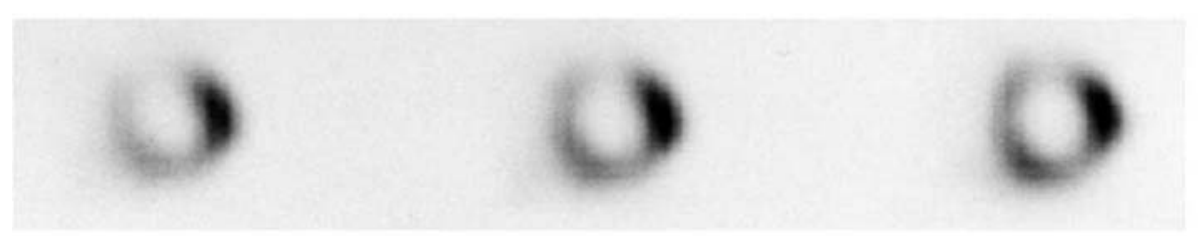

Stress thallium-201 SPECT

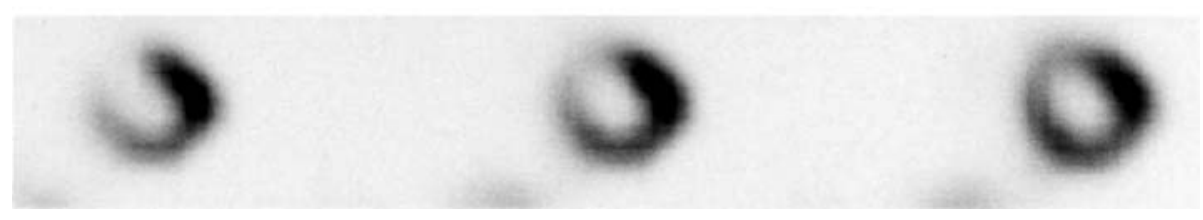

BMIPP SPECT

Figure 6 Radiolabelled iodinated fatty acid analogue, BMIPP, used to assess fatty acid utilisation in the myocardium. In this example, the top row depicts normal resting perfusion by thallium 201 SPECT imaging, in short axis tomograms. The middle row demonstrates extensive stress induced thallium 201 perfusion abnormalities consistent with inducible ischaemia of the anterior, septal, and inferior walls. In the bottom row, the BMIPP SPECT images of the analogous short axis tomograms, obtained at rest, demonstrate ongoing abnormality in fatty acid metabolism in the anterior wall, septum and inferior wall, temporally distinct from the presenting ischaemic insult. Thus, this technique may provide risk stratification information based on the magnitude of abnormal fatty acid metabolism using rest imaging alone without the need for a stress test. This concept is currently under study. Adapted from Kawai and colleagues, ${ }^{39}$ images provided by Dr Nagara Tamaki, with permission.

sestamibi SPECT imaging after MI in 134 consecutive patients within 14 days of an uncomplicated MI and found that the extent of ischaemia on the SPECT sestamibi scan was the only significant correlate of a future cardiac event on Cox regression analysis (fig 7). Among the subgroup who had received thrombolytic therapy $(40 \%)$, the extent of sestamibi SPECT ischaemia remained a strong correlate of a cardiac event. Mahmarian and colleagues ${ }^{46}$ found that the quantitated extent of ischaemia on adenosine SPECT thallium 201 imaging was an important predictor of post-MI cardiac events by Cox regression analysis.

These data are likely representative of the contemporary management of MI in large populations and suggest that there is an important role for scintigraphic imaging in the current era. In a study also likely to be reflective of current practice in the UK, Basu and colleagues studied 100 patients after uncomplicated MI who had received thrombolytic therapy and were admitted to the critical care unit at Northwick Park Hospital, and subsequently underwent stress

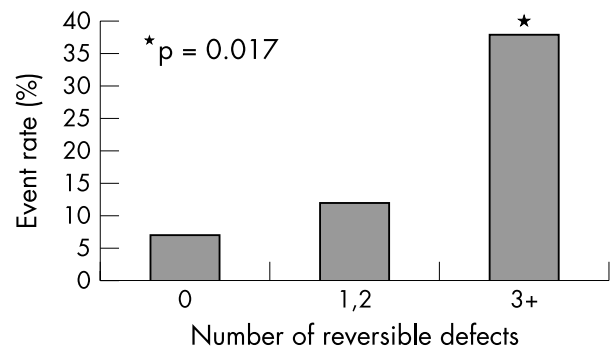

Figure 7 Relation between the post-Ml extent of ischaemia las determined by the number of perfusion defects by SPECT Tc $99 \mathrm{~m}$ sestamibi) with cardiac event rate (y axis). Patients with more extensive ischaemia are at progressively higher risk of unfavourable outcome $(p=0.017)$ compared with patients with no reversible defects. From Travin and colleagues. ${ }^{45}$ thallium 201 imaging. ${ }^{47}$ When information regarding ischaemia was obtained by the use of stress and nitrate enhanced rest thallium 201 imaging, reversible ischaemic defects were detected in 68 patients, of whom $49 \%$ had events, while of 32 without evidence of inducible ischaemia, only $13 \%$ had subsequent cardiac events (hazard ratio 8.1 , 95\% CI 2.7 to 23.8; $\mathrm{p}<0.001)$.

\section{Clinical trials incorporating results of stress testing following MI}

The true power of a predictive test is best demonstrated, insofar as it can be used for clinical decision making, to improve outcomes not only predict outcomes. In this regard, several studies have been reported in which the presence of inducible ischaemia following MI was used to guide clinical decisions.

The TIMI-II trial ${ }^{48}$ randomised 3339 patients, who received intravenous tissue-type plasminogen ( $\mathrm{t}-\mathrm{PA}$ ) for acute MI, to either an invasive strategy (cardiac catheterisation at 18-48 hours after infarction with subsequent angioplasty or bypass surgery depending on anatomy), or a conservative arm in which cardiac catheterisation was performed only in response to spontaneous or inducible ischaemia (by stress radionuclide ventriculography). The one year outcome results were similar between the invasive or conservative strategy. The investigators concluded that a non-invasive strategy with pre-discharge stress imaging examining for the presence of inducible ischaemia will be associated with similar outcomes with less need for catheterisation and revascularisation than a direct catheterisation strategy. Cost effectiveness is implied by the similar outcomes associated with fewer catheterised patients.

The absence of scintigraphic ischaemia has also been investigated as to its influence on clinical decision making for intervention after MI. Ellis and colleagues ${ }^{49}$ reported on 87 patients who had received thrombolytic therapy for acute MI 
and subsequently had a negative functional test for ischaemia in the setting of a residual stenosis of the infarct related artery. The patients were randomised to either medical therapy or to angioplasty of the infarct related artery stenosis. There were no differences between the study groups in the change from rest to exercise ejection fraction or in the resting ejection fraction at the six week end point. Actuarial 12 month infarct-free survival was $98 \%$ in the conservative therapy group and $91 \%$ in the group randomised to percutaneous coronary intervention $(\mathrm{p}=0.07$ ) (fig 8). This trial demonstrates that patients with no evidence of scintigraphic ischaemia within the infarct zone, even in the setting of a residual stenosis of the infarct related artery, derive no benefit from angioplasty of the infarct related artery.

The results of such trials suggest that scintigraphic testing for the presence and extent of myocardial ischaemia in the aftermath of an acute MI can indeed play an important role in clinical decision making regarding the need for and utility of catheterisation and revascularisation, and can also identify a cohort of patients whose outcome will be favourable without catheterisation. With the expectation of wider application of aggressive secondary prevention strategies in the post-MI population, it might be anticipated that the low risk post-MI outcome cohort will continually expand, making their identification before discharge even more compelling.

\section{Very early post-MI risk stratification}

Pharmacologic stress with adenosine or dipyridamole induces coronary hyperaemia with only minimal increments in oxygen demand, thus is potentially safer to administer very early after MI. This concept, which if feasible would potentially allow identification of high and low risk cohorts earlier in the post-MI recovery phase than standard stress testing, was examined by Brown and colleagues. ${ }^{50}$ In this study, 451 patients were randomised to a standard post-MI evaluation strategy, or to a strategy incorporating dipyridamole sestamibi imaging 2-3 days after uncomplicated MI. The testing was safe, ${ }^{51}$ and the imaging information supplied powerful risk stratification data predicting two year outcomes, that more powerfully predicted outcomes than the submaximal stress imaging data. The investigators concluded

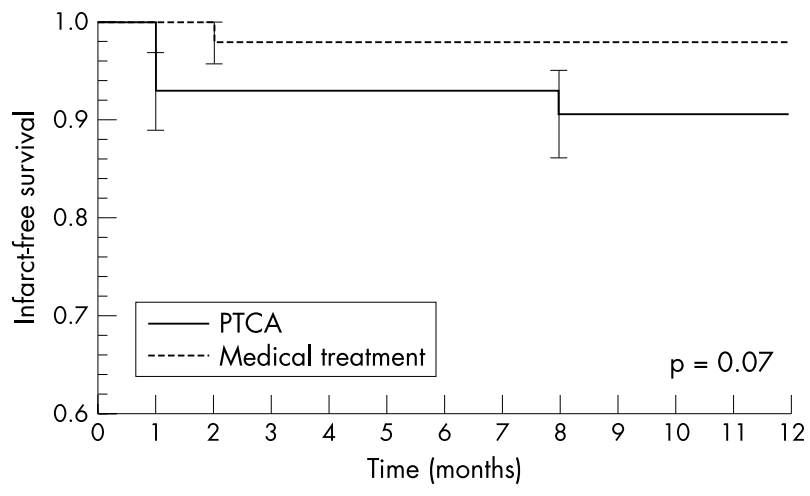

Figure 8 In a study by Ellis and colleagues, patients who had received thrombolytic therapy for acute MI who had a residual stenosis of the infarct related artery but no inducible ischaemia in the infarct territory by MPI were randomised to either a strategy of percutaneous transluminal coronary angioplasty (PTCA) of the residual stenosis or a strategy of no PTCA. Shown is a plot of actuarial freedom from cardiac events after randomisation to PTCA (solid line) or medical therapy (dashed line). There is no difference in outcome between the groups. Hence, identification of inducible ischaemia or lack thereof within the infarct zone by perfusion imaging after acute $\mathrm{Ml}$ and reperfusion therapy can guide management decisions regarding revascularisation strategy. In the absence of any residual infarct zone ischaemia, there appears little benefit from a strategy of revascularisation. From Ellis and colleagues. ${ }^{49}$ that, "this technique can allow management decisions to be made earlier with regard to acute MI patients and could have important economic impact if applied widely".

It may be important to separate patients receiving thrombolytic therapy (for whom the decision for coronary revascularisation performed out of the acute phase will be dependent upon the extent of jeopardised/ischaemic myocardium), and patients treated by primary or rescue PTCA. In the latter patients rest/redistribution MPI may be helpful to predict late functional recovery. ${ }^{52} 53$

\section{Studies examining both perfusion imaging and LV function following acute MI}

Large databases have been examined in both the pre- and post-reperfusion era to assess post-MI LV function as a predictor of short and long term outcomes. ${ }^{54-56}$ As post-MI ejection fraction falls, there is a progressive increase in mortality risk. The availability of gated SPECT imaging to simultaneously evaluate myocardial perfusion and LV function at little additional effort and cost, compared to perfusion imaging alone, raises the important question regarding the incremental information provided by combining the analysis of perfusion and function information within one test. As the cost of adding the gated SPECT LV function information is modest once perfusion imaging is performed, the increment of information required for cost effectiveness is similarly modest.

Mahmarian and colleagues ${ }^{46}$ studied 146 post-MI patients with assessment of LV function as well as adenosine SPECT thallium tomography, and related findings to cardiac events over an average of 16 months of follow up (fig 9). Knowledge of both the extent of perfusion defect/ischaemia size along with information on LV ejection fraction allowed risk categorisation superior to that provided by either variable alone. These data strongly suggest that measurement of perfusion abnormalities (total defect size and quantitative extent of ischaemia) and LV ejection fraction following MI have complimentary roles, and together are powerful instruments for categorising patient risk in this setting. Given the opportunity to derive these variables simultaneously, gated

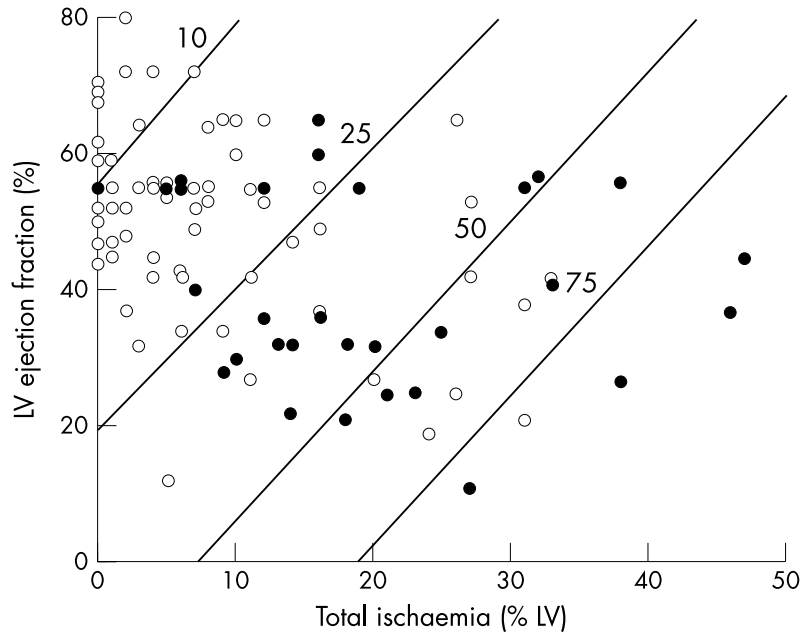

Figure 9 Cox regression models displaying one year post-MI risk for cardiac event according to LV ejection fraction and total LV ischaemia. The diagonal lines are representative of isobars of per cent risk of event. Patient risk for any cardiac event increases as total LV ischaemia increases and LV ejection fraction decreases. LV ejection fraction and scintigraphic results for each of 92 patients who did (solid circles) or did not (open circles) have subsequent cardiac events over entire follow up period are plotted against calculated risk at one year. From Mahmarian and colleagues. ${ }^{46}$ 
A
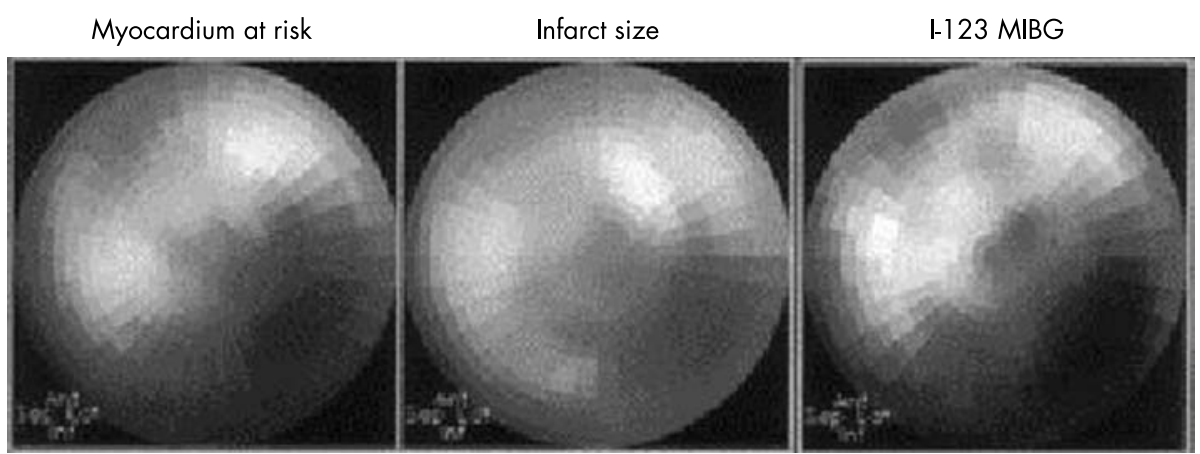

B
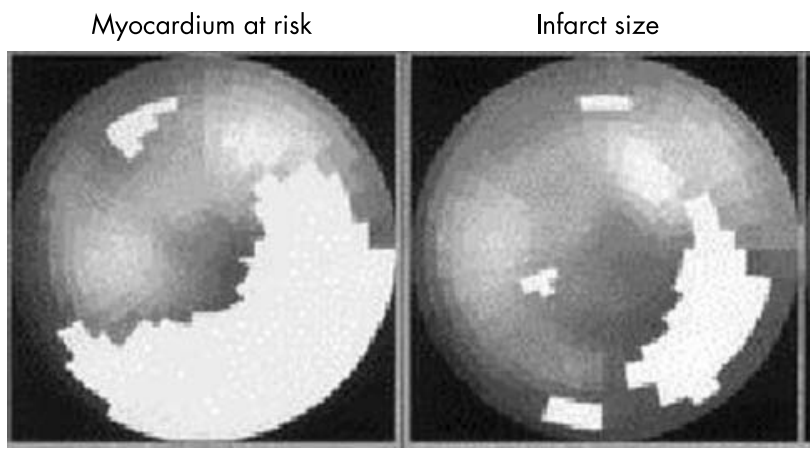

SPECT perfusion imaging should provide a powerful impetus for optimising post-MI risk stratification.

\section{FUTURE DIRECTIONS}

\section{Response of ischaemia to medical therapy}

Among patients with high risk scintigraphic or clinical signs, only a minority will indeed suffer an important cardiac event during follow up. Therefore in order to prevent an exceptional cardiac event, most, if not all, of these high risk patients undergo intervention. While clinicians accept this trade off, recent intriguing data involving perfusion imaging suggest that the response of scintigraphic ischaemia to medical therapy may identify the subgroup within the high risk cohort who will suffer a cardiac event, and would therefore benefit most from an invasive strategy.

Dakik and colleagues ${ }^{57}$ studied 44 stable MI survivors with adenosine SPECT thallium 201 imaging approximately four days after acute MI, who had large total and ischaemic perfusion defect size (that is, a high risk result). Patients
Figure 10 Use of MIBG imaging of cardiac sympathetic innervation. In a study of acute $\mathrm{Ml}$ patients, the territory of sympathetic denervation (MIBG defect, polar map images in right columns in panels $A$ and $B$ )

corresponded more closely to the initial MI risk area (polar map images in left columns) than the final infarct size (polar map images in middle columns). From Matsunari and colleagues. ${ }^{60}$
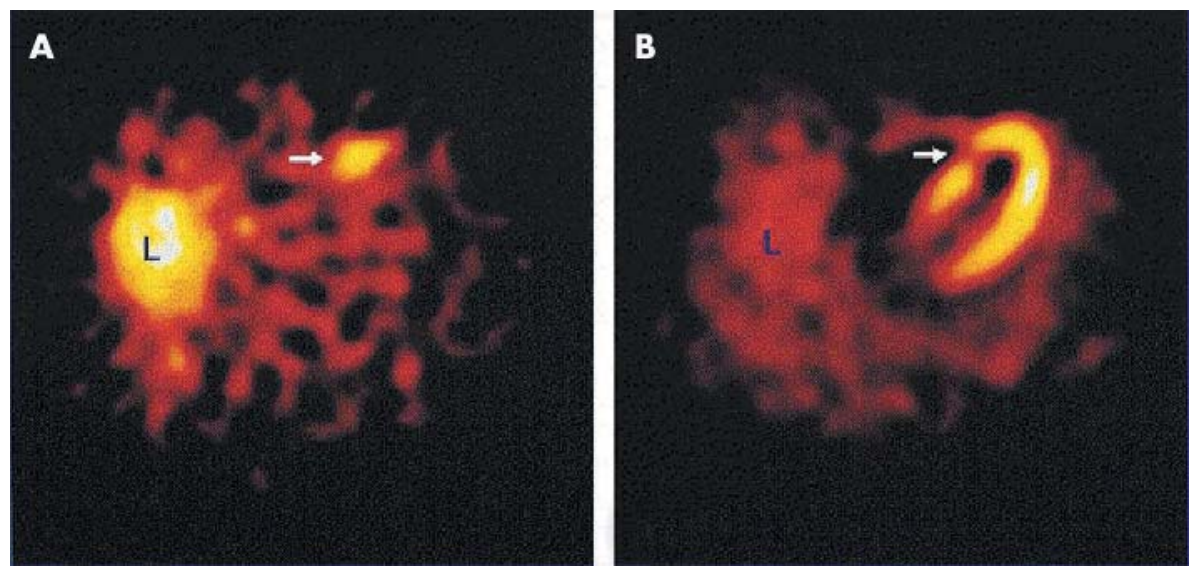

Figure 11 Anteroseptal uptake of Tc $99 \mathrm{~m}$ labelled annexin- $\mathrm{V}$ is seen (left panel) in the territory of a resting perfusion defect consistent with the infarct zone (right panel). Reprinted from Hofstra and colleagues, ${ }^{61}$ with permission. 


\section{Assessment of cardiac sympathetic innervation}

An emerging area of scintigraphic risk stratification in the post-MI setting involves the use of I-123 metaiodobenzylguanidine (MIBG) imaging of cardiac sympathetic innervation. In the post-MI setting, several studies have shown that the territory of abnormal MIBG uptake, corresponding to sympathetic denervation, may often exceed the final infarct size, and that such patients may be at higher risk for subsequent ventricular arrhythmias. Matsunari and colleagues ${ }^{60}$ using SPECT Tc $99 \mathrm{~m}$ sestamibi imaging of infarct risk area and final infarct size, as well as MIBG imaging in acute MI patients, demonstrated that the territory of sympathetic denervation corresponded more closely to the initial MI risk area than the final infarct size (fig 10). Should such findings in the contemporary therapeutic era prove prognostic for late post-MI outcomes, as suggested by earlier studies, MIBG imaging may prove useful in selecting post-MI patients who may optimally benefit from implantable defibrillators.

\section{Annexin imaging}

An exciting new potential approach to evaluating post-MI patients using non-invasive imaging is the in vivo visualisation of apoptosis or programmed cell death. Investigations are now underway in humans using Tc $99 \mathrm{~m}$ labelled annexin- $\mathrm{V}$, which localises to apoptotic cells. Hofstra and colleagues $^{61}$ found positive uptake of this agent in six out of seven post-MI patients, localised to areas of resting perfusion defects (fig 11). Should these data be confirmed in larger studies, it will herald the onset of the ability to noninvasively track this process in clinical syndromes, and potentially study therapeutic approaches to attenuate the unfavourable pathophysiology.

\section{CONCLUSIONS}

Radionuclide imaging can supply critically important prognostic information in the setting of ACS, whether in suspected ACS without obvious ischaemic ECG changes in the CPAU or ED, or in the clinical setting of NSTEMI/UA, or STEMI. The high negative predictive value in ED patients with chest pain and non-diagnostic ECG changes can drive triage decisions regarding admission or discharge. Among ACS patients who have stabilised clinically but have a high risk substrate identified by imaging, outcomes may be improved by revascularisation. The absence of ischaemia on perfusion imaging in the aftermath of a clear ACS suggests that a conservative management strategy, including aggressive secondary prevention medical measures, is appropriate. Ongoing research in this area involves new tracers of metabolic and pathophysiological processes that may allow more specifically directed therapy after ACS.

\section{Authors' affiliations}

J E Udelson, Division of Cardiology, Tufts-New England Medical Center Hospitals, Tufts University School of Medicine, Boston, Massachusetts, USA

E J Flint, Dudley Group of Hospitals, Wordsley Hospital, Stourbridge, West Midlands, UK

Correspondence to: Dr Jane Flint, Dudley Group of Hospitals, Wordsley Hospital, Stourbridge, West Midlands, UK; jane.flint@dudleygoh-tr. wmids.nhs.uk

\section{REFERENCES}

1 Department of Health (2000). National service framework for coronary heart disease http://ww.doh.gov.uk/nsf/coronary.htm.

2 Underwood SR, Godman B, Salyani, et al. Economics of myocardial perfusion imaging in Europe-EMPIRE Study. Eur Heart J 1999;20:157-66.

3 British Cardiac Society Guidelines, and Medical Practice Committee and Royal College of Physicians Clinical Effectiveness and Evaluation Unit (2001).
Guidelines for the management of patients with acute coronary syndromes without persistent ECG ST segment elevation. Heart 2001;85:133-42.

4 Farkouh ME, Smars P, Reeder GS, et al. A clinical trial of a chest pain observation unit for patients with unstable angina. N Eng J Med 1998:339:1882-8.

5 Goodacre SW. Should we establish chest pain observation units in the UK? A systematic review and critical appraisal of the literature. J Accid Emerg Med 2000;17(S): 1-6.

6 Stratmann HG, Younis LT, Wittry MD, et al. Exercise technetium-99m myocardial tomography for the risk stratification of men with medically treated unstable angina pectoris. Am J Cardiol 1995;76:235-40.

7 Stratman HG, Tamesis BR, Younis LT, et al. Prognostic value of predischarge dipyridamole technetium sestamibi myocardial tomography in medically treated patient with unstable angina. Am Heart J 1995;130:734-40.

8 Wackers FJT, Lie KI, Liem KL, et al. Potential value of thallium-201 scintigraphy as a means of selecting patients for the coronary care unit $\mathrm{Br}$. Heart $\mathrm{J}$ 1979:41:111-7.

9 Beller GA, Radiopharmaceuticals in nuclear cardiology In: Beller GA, eds. Clinical nuclear cardiology. Philadelphia: Saunders, 1995:37-81.

10 Bilodeau L, Theroux P, Gregoire J, et al. Technetium-99m sestamibi tomography in patients with spontaneous chest pain: correlations with clinical electrocardiographic and angiographic findings. J Am Coll Cardiol 1991;18:1684-91

11 Christian TF, Clements IP, Gibbons RJ, et al. Non-invasive identification of myocardium at risk in patients with acute myocardial infarction and non diagnostic electrocardiograms with technetium- $99 \mathrm{~m}$ sestamibi. Circulation 1991;83:1615-20.

12 Varetto T, Cantalupi D, Altieri A, et al. Emergency room technetium-99m sestamibi imaging to rule out acute myocardial ischaemic events in patients with non diagnostic electrocardiography. J Am Coll Cardiol 1993;22:1804-8

13 Hilton TC, Thompson RC, Williams $\mathrm{H}$, et al. Technetium- $99 \mathrm{~m}$ sestamibi myocardial perfusion imaging in the emergency room evaluation of chest pain. J Am Coll Cardiol 1994;23:1016-22.

14 Tatum JL, Jesse RI, Kontos MC, et al. Comprehensive strategy for the evaluation and triage of the chest pain patient. Ann Emerg Med 1997;29:116-25

15 Heller GV, Stowers SA, Hendel RC, et al. Clincal value of acute rest technetium-99m tetrofosmin tomographic myocardial perfusion imaging in patients with acute chest pain and non diagnostic electrocardiogram. J Am Coll Cardiol 1998;31:1011-7.

16 Kontos MC, Jesse RL, Anderson P, et al. Comparison of myocardial perfusion imaging and cardiac troponin I in patients admitted to the emergency department with chest pain. Circulation 1999;99:2073-8.

17 Stowers SA, Eisenstein EL, Wackers FJ, et al. An economic analysis of an aggressive diagnostic strategy with single photonemission computed tomography myocardial perfusion imaging and early exercise stress testing in emergency department patients who present with chest pain but non diagnostic electrocardiograms: results from a randomized trial. Ann Emerg Med 2000;35:17-25.

18 Udelson JE. The emergency room assessment of sestamibi for the evaluation of chest pain trial. Proceedings of the $72^{\text {nd }}$ American Heart Association. Atlanta: American Heart Association, 1999.

19 Braunwald E, Antman EM, Beasley JW, et al. ACC/AHA Guidelines of the management of patients with unstable angina and non ST segment elevation myocardial infarction: executive summary and recommendations: a report of the ACC/AHA taskforce on practice guidelines. Circulation 2000;102:1193-209.

20 Ryan TJ, Antman EM, Brooks NH, et al. ACC/AHA guidelines for the management of patients with acute myocardial infarction: executive summary and recommendations. A report of the American College of Cardiology/ American Heart Association. Circulation 1999;100:1016-30.

21 Joint Royal College of Physicians/British Cardiac Society. Fifth report on the provision of services for patients with heart disease. Heart 2002;88(suppl III):iii1-iii59.

22 Ziffer JA, Nateman DR, Janowitz WR, et al. Improved patient outcomes and cost effectiveness of utilizing nuclear cardiology protocols in an emergency department chest pain centre. J Nucl Med. 1998;39: 13 [astract]).

23 Braunwald EB, et al. Unstable Angina Clinical Practice Guideline Number 10. Agency for Health Care Policy and Research publication No. 94-0602, 1994

24 Brown KA. Management of unstable angina: the role on noninvasive risk stratification. J Nucl Cardiol 1997;4:S164-8.

25 Klocke FJ, Baird MG, Lorell BH, et al. Guidelines for clinical use of cardiac radionuclide imaging. J Am Coll Cardiol 2003;42:1318-33.

26 The TIMI-III-B investigators. Effects of tissue plaminogen activator and a comparison of early invasive and conservative strategies in unstable angina and non-Q-wave myocardial infarction: Results of the TIMI-III B Trial Thrombolysis and Myocardial Ischaemia. Circulation 1994;89:1545-56.

27 Boden WE, O'Rourke RA, Crawford MH, et al. Outcomes in patients with acute non- $Q$-wave myocardial infarction randomly assigned to an invasive as compared to a conservative management strategy: Veteran affairs non-Qwave infarction strategies in hospital (VANQUISH) trial investigators. N Engl J Med 1998;338:1785-92.

28 FRISC II Investigators. Invasive compared with non invasive treatment in unstable coronary artery disease: FRISC-II prospective randomized multicentre study. Lancet 1999;354:708-15.

29 Cannon CP, Weintraub WS, Demopoulos LA, et al. Comparison of early invasive and conservative strategies in patients with unstable coronary syndromes treated with the glycoprotein Ilb/Illa inhibitor tirofiban. NEngl J Med 2001;344: 1879-87. 
30 Barnett PG, Cheo S, Boden WE, et al. Cost-effectiveness of a conservative, ischaemia guided management strategy after non- $Q$-wave myocardial infarction: results of a randomized trial. Circulation 2002; 105:680-4.

31 Goyal A, Samaha FF, Boden WE, et al. Stress test criteria used in the conservative arm of FRISC-II underdetects surgical coronary artery disease when applied to patients in the VANQWISH trial. J Am Coll Cardiol 2002;39:1601-7.

32 Mark DB, Lee TH. Conservative management of acute coronary syndrome. Cheaper and better for you? Circulation 2002;105:666-8.

33 Mehta S, Yousuf S, Hunt D, et al. Invasive versus conservative management of unstable angina and non Q-wave myocardial infarction: a meta-analysis. Circulation 1999;100:1-775.

34 Dargie H. Myocardial infarction: redefined or reinvented? Heart 2002;88:1-3.

35 Solomon DH, Stone PH, Glynn RJ, et al. Use of risk stratification to identify patients with unstable angina likeliest to benefit from an invasive versus conservative management strategy. J Am Coll Cardiol. 2001;38: 969- 76)

36 Antman EM, Cohen M, Bernink PJ, et al. The TIMI risk score for unstable angina/non-ST elevation Ml: a method for prognostication and therapeutic decision making. JAMA 2000;284:835-42.

37 Parisi AF, Kuri S, Deupree RH, et al. Medical compared with surgical management of unstable angina pectoris: five year mortality and morbidity in the Veterans Administration study. Circulation 1989;80:1176-89.

38 Jafary F, Udelson JE. Assessment of myocardial perfusion and left ventricular function in acute coronary syndromes: implications for gated SPECT imaging. In:Germano and Berman. Cardiac gated SPECT imaging 1999.

39 Kawai Y, Tsukamoto E, Nozaki Y, et al. Significance of reduced uptake of iodinated fatty acid analogues for the evaluation of patients with acute chest pain. J Am Coll Cardiol 2001:38:1888-94.

40 Gibson RS, Watson DD, Craddock GB, et al. Prediction of cardiac events after uncomplicated myocardial infarction: prospective study comparing pre discharge exercise thallium-201 scintigraphy and coronary angiography. Circulation 1983:68:321-6.

41 Volpi A, deVita C, Franzosi MG, et al. Predictors of nonfatal reinfarction in survivors of myocardial infarction after thrombolysis. Results of the Gruppo Italiano per lo Studio della Sporavvivenza nell 'Infarto Moocardico (GISSI-2) J Am Coll Cardiol 1994;24(3):608-15.

42 Leppo JA, O'Brien J, Rothendler JA, et al. Dipyridamole-thallium-201 scintigraphy in the prediction of future cardiac events after acute myocardial infarction. N Engl J Med 1984;310:1014-8.

43 Gimple LW, Hutter AM Jr, Guiney TE, et al. Prognostic utility of predischarge dipyridamole-thallium imaging compared to predischarge submaximal exercise electrocardiography and maximal exercise thallium imaging after uncomplicated acute myocardial infarction. Am J Cardiol 1989;64:1243-8.

44 Younis LT, Byers S, Shaw L, et al. Prognostic value of intravenous dipyridamole thallium scintigraphy after an acute myocardial ischemic event. Am J Cardiol 1989;64:161-6.

45 Travin MI, Dessovki A, Cameron T, et al. Use of exercise technetium- $99 \mathrm{~m}$ sestamibi SPECT imaging to detect residual ischaemia and for risk stratification after acute myocardial infarction. Am J Cardiol 1995;75:665-9.

46 Mahmarian JJ, Mahmarian AC, Marks GF, et al. Role of adenosine thallium 201 tomography for defining long term risk in patients after acute myocardial infarction. J Am Coll Cardiol 1995;25:1333-40.
47 Basu S, Senior R, Raval U, et al. Superiority of nitrate-enhanced ${ }^{201} \mathrm{Tl}$ over conventional redistribution ${ }^{201} \mathrm{Tl}$ imaging for prognostic evaluation after myocardial infarction and thrombolysis. Circulation 1997;96:2932-7.

48 TIMI Study Group. Comparison of invasive and conservative stategies after treatment with intravenous TPA in acute myocardial infarction: results of the thrombolysis in MI (TIMI) phase II trial. NEng J Med 1989;320:618-27.

49 Ellis SG, Mooney MR, George BS, et al. Randomised trial of late elective angioplasty versus conservative management for patients with residual stenoses after thrombolytic treatment for myocardial infarction (TOPS Trial). Circulation 1992;86:1400-6.

50 Brown KA, Heller GV, Landin RS, et al. Early dipyridamole ${ }^{99 \mathrm{~m} T c-s e s t a m i b i}$ single photon emission computed tomographic imaging 2 to 4 days after acute myocardial infarction predicts in-hospital and postdischarge cardiac events: comparison with submaximal exercise imaging. Circulation 1999; 100:2060-6.

51 Heller GV, Brown KA, Landin RJ, et al. Safety of early intravenous dipyridamole technetium 99m sestamibi SPECT myocardial perfusion imaging after uncomplicated first myocardial infarction. Am Heart $J$ 1997;134:105-11.

52 Takeishi Y, Sukekawa H, Fugiwira S, et al. Reverse redistribution of Tc- $99 \mathrm{~m}$ sestamibi following PTCA in acute myocardial infarction. J Nucl Med 1996;37(8): 1289-94

53 Leoncini $M$, Sciagra R, Bellandi $F$, et al. Low-dose dobutamine in late enhanced Tc-99m Sestamibi gated SPECT versus low-dose dobutamine echocardiography in detecting reversible dysfunction in ischaemic cardiomyopathy. J Nucl Cardiol 2002:9, 4:402-6.

54 The Multicenter Postinfarction Research Group. Risk stratification and survival after myocardial infarction. N Engl J Med 1983;309:331-6.

55 Zaret BL, et al. Value of radionuclide rest and exercise left ventricular ejection fraction in assessing survival of patients after thrombolytic therapy for acute myocardial infarction: results of thrombolysis in myocardial infarction (TIMI) phase II study. J Am Coll Cardiol 1995;26:73-9.

56 Volpi A, deVita C, Franzosi MG, et al. Determinants of 6-month mortality in survivors of myocardial infarction after thrombolysis. Results of the Gruppo Italiano per lo Studio della Sporavvivenza nell 'Infarto Moocardico (GISSI-2) database. Circulation 1993;88:416-29.

57 Dakik HA, Kleiman NS, Farmer JA, et al. Intensive medical therapy versus coronary angioplasty for suppression of myocardial ischaemia in survivors of acute myocardial infarction: A prospective randomized pilot study. Circulation 1998;98:2017-23.

58 Sridhara BS, Dudzic E, Basu S, et al. Reverse redistribution of thallium-201 represents a low risk thrombolysed patient following myocardial infarction. Eur J Nucl Med 1994;21:1094-7.

59 Sharir T, Germano G, Kavanagh PB, et al. Incremental prognostic value of post-stress left ventricular ejection fraction and volume by gated myocardial perfusion single photon emission computed tomography. Circulation 1999; 100:1035-42.

60 Matsunari I, Schricke U, Bengel FM, et al. Extent of cardiac sympathetic neuronal damage is determined by the area of ischaemia in patients with acute coronary syndromes. Circulation 2000;101:2579-85.

61 Hofstra L, Liem IH, Dumont EA, et al. Visualization of cell death in vivo in patients with acute myocardial infarction. Lancet 2000;356:209-12. 